\title{
REMOCIÓN DE IONES COBRE Y NIQUEL CON CÁSCARA DE MANÍ
}

\author{
P. TAPIA ${ }^{1}$, O. PAVEZ ${ }^{1,2}$, N. GARRIDO ${ }^{1}$, B. SEPÚLVEDA ${ }^{2}$ \\ ${ }^{1}$ Departamento de Ingeniería en Metalurgia, ${ }^{2}$ Centro Regional de Investigación y Desarrollo Sustentable de \\ Atacama/ Universidad de Atacama, Chile. \\ bernardo.sepulveda@uda.cl $\left.\right|^{2}$
}

Submetido 07/03/2018 - Aceito 27/08/2018

DOI: $10.15628 /$ holos.2018.7064

\section{RESUMEN}

En el presente trabajo se estudió la remoción de iones cobre y níquel utilizando cáscaras de maní mediante pruebas en lote en sistemas monocomponente y bicomponente (pruebas de remoción simultánea). En este estudio se evaluó el efecto de las variables $\mathrm{pH}$, tiempo de contacto, concentración de biosorbente, concentración del ión metálico. El resultado mostró que las cáscaras de maní fueron un biosorbente relativamente efectivo para la eliminación de estos iones, alcanzándose remoción que varió entre $4,3 \%$ y $68,3 \%$ para iones cobre y entre $3,6 \%$ y $47,6 \%$ para iones níquel. La máxima remoción de los iones cobre y níquel se alcanzó en pH 3. La cinética de adsorción de ambos iones se produce rápidamente durante los primeros 15 minutos lográndose eliminaciones cercanas a 60,0 \% para el cobre y 43,0 \% para el níquel, no obstante, los valores máximos de adsorción se alcanzan a los 30 minutos (cobre) y 60 minutos (níquel). Se observó que a medida que aumenta la concentración del biosorbente la eliminación de los iones cobre y níquel disminuye levemente. Las pruebas de remoción simultánea mostraron que la eliminación de iones níquel $(45,7 \%)$ fue inferior que la de iones cobre $(69,9 \%)$, estos resultados fueron muy similares a los alcanzados en las pruebas individuales de remoción de ambos iones.

PALABRAS CLAVE: Remoción, iones cobre, iones níquel, cáscaras de maní, biosorción

\section{REMOVAL OF COPPER AND NICKEL IONES WITH PEANUT SHELL}

\begin{abstract}
In the present work, the removal of copper and nickel ions using peanut hulls by batch tests in monocomponent and bi-component systems (simultaneous removal tests) was studied. This study evaluated the effect of $\mathrm{pH}$, contact time, biosorbent concentration and metal ion concentration. The results showed that the peanut hulls were a relatively effective biosorbent in the removal of ions, obtaining removals between $4.3 \%$ to $68.3 \%$ for copper ions and $3.6 \%$ to $47.6 \%$ for nickel ions. The maximum removal of copper and nickel ions was reached at $\mathrm{pH} 3$. Sorption kinetics of both ions occurs rapidly during the first 15 minutes with eliminations
\end{abstract}

close to $60.0 \%$ for copper and $43.0 \%$ for nickel, however, maximum adsorption values are reached after 30 minutes (copper) and 60 minutes (nickel). It was observed that as the concentration of the biosorbent increases the elimination of the copper and nickel ions decreases slightly. Simultaneous removal tests showed that the removal of nickel ions (45.7\%) was lower than of copper ions (69.9\%), these results were very similar to those achieved in the individual removal test of both ions.

KEYWORDS: Removal, copper ions, nickel ions, peanut hulls, biosorption. 


\section{INTRODUCCION}

Los metales pesados, entre los diferentes contaminantes existentes, han recibido una especial atención debido a que son elementos tóxicos y contaminantes no biodegradables, que son liberados en el ambiente principalmente por actividades mineras y agrícolas. En el tratamiento de efluentes líquidos, los métodos aplicados para la eliminación de metales pesados son precipitación química, coagulación, intercambio iónico, utilización de membranas, entre otros. Estos procesos en algunos casos resultan poco adecuados debido a la complejidad de su aplicación técnica y al costo económico del tratamiento.

La utilización de la biosorción en el tratamiento de efluentes contaminados con metales pesados se ha convertido en un método alternativo a los tratamientos convencionales, debido a que en general es económicamente factible, sencilla, eficaz y ecológica. En la remoción de metales pesados se han aplicado diferentes biosorbentes, tales como residuos y desechos agrícolas, residuos de fibras de cocos, cáscaras de coco, cáscaras de arroz, cáscaras de maní, cáscaras de nuez, cáscaras de fruta, semillas, algas de diferentes colores, hongos, entre otros.

En la remoción de iones cobre y níquel, desde soluciones acuosas, se ha aplicado diferentes tipos de biosorbentes. Khan et al. (2015) determinaron el efecto de factores que influyen en la adsorción; tales como pH inicial, temperatura, tamaño de partícula y tiempo de contacto; evaluaron la eliminación de $\mathrm{Ni}$ (II) y $\mathrm{Cu}$ (II) utilizando como adsorbentes biomasa pura y químicamente pretratada de Arachis hypogea (cáscaras de maní), Prunus amygdalus (cáscaras de almendra), Arundo donax (caña gigante). Los adsorbentes pretratados aumentaron significativamente su capacidad de adsorción de los metales, lo que indicó que la afinidad entre el metal y el sorbente puede incrementarse después del pretratamiento. Oliveira et al. (2010) investigaron la remoción de $\mathrm{Cu}$ (II), Ni (II) y Zn (II) desde soluciones acuosas utilizando cáscaras de maní (biosorbente); caracterizándolas químicamente y realizando estudios cinéticos, entre otros. Los estudios cinéticos indicaron que la mayor parte de la sorción ocurrió en los primeros 30 minutos para todos los sistemas. En general, se siguió una cinética de pseudo segundo orden, tanto en sistemas monocomponente como de tres componentes. Las cáscaras de maní mostraron una mayor afinidad por el cobre que por el níquel y el zinc cuando ambos están presentes (tricomponente). Oliveira et al. (2009) investigaron el uso de cáscaras de maní en la extracción de cobre y plomo a través de estudios cinéticos. En sistemas de dos componentes se verificó la sorción competitiva de $\mathrm{Cu}$ (II) y $\mathrm{Pb}(\mathrm{II})$. El equilibrio se alcanzó después de 200 min de contacto a 400 rpm de agitación, lográndose en el sistema monocomponente la eliminación del $78 \%$ y $58 \%$ de $\mathrm{Cu}(\mathrm{II})$ y $\mathrm{Pb}(\mathrm{II})$ respectivamente. La mayoría de la remoción de los metales ocurrió en los primeros 20 minutos de contacto. Cáscaras de maní también fueron utilizadas en la eliminación de Cu(II) por Zhu et al. (2009), quienes encontraron que la remoción fue dependiente del pH, alcanzándose un máximo en pH 5,5.

Singh et al. (2013) estudiaron cuatro combinaciones diferentes, mezclando diversas cantidades de biomasa en polvo de Trifolium alexandrinum, polvo de cáscara de Arachis hypogea y aserrín de Eucalyptus cameldulensis, para la eliminación de $\mathrm{Ni}^{2+}, \mathrm{Cu}^{2+}$ y $\mathrm{Zn}^{2+}$ desde soluciones acuosas. La cinética de adsorción de los tres metales alcanzó el equilibrio en 300 min y se describió mejor por 
una cinética de pseudosegundo orden. Estos investigadores concluyeron que los adsorbentes utilizados en diferentes combinaciones son atractivos y opciones alternativas para la eliminación del metal pesado de efluentes industriales.

Kondo et al. (1996) estudiaron la adsorción de iones metálicos en quitosan químicamente modificado por D-galactosa, ellos indicaron que el orden de adsorción de los iones metálicos en este material fue $\mathrm{Cu}>\mathrm{Ni}>\mathrm{Co}$. Recientemente, Candan et al. (2017) informaron el uso de líquenes no vivos, Ramalina fraxinea, como biosorbente para la eliminación de iones CU (II) y Ni (II) desde solución acuosa. Los estudios se realizaron en sistema por lotes investigando los parámetros $\mathrm{pH}$, tiempo de contacto y concentración inicial de los iones metálicos. Estos investigadores evaluaron los datos cinéticos de biosorción con modelos cinéticos de pseudo primer orden, pseudo segundo orden y difusión intrapartícula. Para la eliminación de plomo (II), cobre (II), cadmio (II) y níquel (II), Hawari y Milligan (2006) investigaron la viabilidad del uso de gránulos anaeróbicos, un nuevo tipo de biosorbente consistente en agregados microbianos con una estructura fuerte, compacta y porosa, de excelente capacidad de sedimentación.

Villaescusa et al. (2004) investigaron la utilidad de los desechos de tallos de uva para la eliminación de iones de cobre y níquel desde soluciones acuosas, para ello estudiaron la influencia del $\mathrm{pH}$, del cloruro de sodio y de la concentración de los metales. Estos investigadores indicaron que el proceso de sorción fue relativamente rápido y se alcanzó el equilibrio después de aproximadamente 60 minutos de contacto. Chen et al. (2008) estudiaron la biosorción de iones de níquel y cobre a partir de solución acuosa utilizando biomasa de alga tratada Undaria pinnatifida. Estos investigadores observaron un aumento en la capacidad específica de sorción del metal con el aumento en la concentración inicial de los iones. La óptima condición del valor de pH para el níquel y el cobre fue 4,7 y 4,0 respectivamente, mientras que el tiempo de contacto fue de aproximadamente 100 minutos.

Sag et al. (2000) estudiaron la biosorción de tres iones metálicos divalentes [Pb (II), Ni (II) y Cu (II)] utilizando Rhizopus arrhizus, un hongo filamentoso, en un sistema agitado de lote. Los datos de biosorción multimetal se evaluaron en términos de isotermas de equilibrio y rendimientos de adsorción. La capacidad de biosorción de $\mathrm{Pb}$ (II) en todos los sistemas estudiados fue mayor que la de los iones $\mathrm{Ni}$ (II) y $\mathrm{Cu}$ (II). Las capacidades relativas fueron $\mathrm{Pb}$ (II)> Ni (II)> Cu (II) a pH 5,0 en sistemas simples, binarios y ternarios.

Kumar et al. (2012) discutieron sobre la adsorción de los iones metálicos Cu (II), Cd (II), Zn (II) y $\mathrm{Ni}$ (II) en cáscara de nuez a partir de una solución acuosa preparada con ácido sulfúrico. Esos investigadores indicaron que el proceso de adsorción depende del pH de la solución, de la dosis de adsorbente, del tiempo de contacto, de la concentración inicial de los iones metálicos y de la temperatura. La cinética de la adsorción de los iones metálicos sobre el adsorbente fue relativamente rápida, siguió un modelo cinético de pseudo segundo orden y el equilibrio se alcanzó a los 30 minutos. La biosorción de cobre (II), zinc (II), cadmio (II) y plomo (II) en biomasa de avena fatua a partir de soluciones acuosas y el efecto de estos metales en el crecimiento de esta avena silvestre fue investigado por Areco et al. (2013). Estos investigadores informaron que la cinética de adsorción de todos los metales siguió un modelo de pseudo segundo orden y que el crecimiento de 
la avena fatua se vio afectado por la presencia de todos los metales. La disminución en la tasa de crecimiento con el aumento de la concentración de metal fue más notoria para el zinc. Una investigación para explorar el uso de cáscaras de fruta (Adononsia digitata) en la eliminación de iones de plomo (II) y cobre (II) a partir de soluciones acuosas fue desarrollada por Chigondo et al. (2013), quienes realizaron experimentos por lotes para determinar el efecto de la variación de parámetros de adsorción en la eliminación de estos iones. Estos investigadores informaron que la adsorción de $\mathrm{Pb}$ (II) era máxima a pH 5 utilizando una dosis de adsorbente de $0,7 \mathrm{~g}$ y que la adsorción de $\mathrm{Cu}$ (II) era óptima a pH 6 usando una dosis de adsorbente de 0,9 g. Concluyeron que las cáscaras de fruta estudiadas se pueden utilizar como un adsorbente rentable para la eliminación de iones plomo (II) y cobre (II) desde soluciones acuosas en el tratamiento de efluentes industriales.

Acheampong et al. (2012) investigaron la cinética de adsorción de Cu (II) sobre cáscaras de coco y semillas de Moringa oleifera mediante técnicas de lotes, encontrando que los datos cinéticos se describían mejor mediante el modelo de pseudo segundo orden. Los resultados obtenidos indicaron que la tasa de sorción era controlada por la difusión intrapartícula, sin embargo, la difusión de la película debe considerarse, especialmente en la etapa inicial de la sorción.

En el presente trabajo se realiza un estudio, a escala de laboratorio, sobre la remoción de iones cobre y níquel en soluciones acuosas utilizando como biosorbente cáscaras de maní y se evalúa el efecto de las variables $\mathrm{pH}$, tiempo de contacto, concentración de biosorbente y concentración del ión metálico. Se utiliza cáscaras de maní por ser un material residual abundante, blando, fácil de obtener, y teniendo las características que se indican en bibliografía, resulta interesante usarlo en la eliminación de metales a bajo costo. En el desarrollo de este trabajo se contemplan dos etapas, en la primera parte se evalúa la remoción individual de los iones cobre y níquel (sistema monocomponente) y en una segunda etapa se realiza la remoción simultánea de ambos iones (sistema bicomponente).

\section{METOdOLOGÍA}

Las pruebas experimentales contempladas en este estudio se realizaron en el Laboratorio de Tratamiento de Efluentes del Departamento de Ingeniería Metalúrgica de la Universidad de Atacama, mientras que los análisis químicos fueron efectuados en el Instituto de Investigaciones Científicas y Tecnológicas (IDICTEC), de la misma Universidad.

\subsection{Preparación del biosorbente}

Cáscaras de maní fueron utilizadas como biosorbente, las cuales inicialmente se secaron en una estufa marca Memmert a una temperatura de $60^{\circ} \mathrm{C}$ y posteriormente se redujeron de tamaño hasta alcanzar una granulometría $100 \%-1 / 2$ pulgada. A continuación, las cáscaras fueron homogeneizadas obteniéndose finalmente las muestras de biosorbente para la investigación. Las cáscaras de maní presentaron una densidad aparente de $109,2 \mathrm{~kg} / \mathrm{m}^{3}$. 


\subsection{Efluentes y reactivos}

Efluentes artificiales fueron preparados en un vaso de precipitado de $600 \mathrm{~mL}$ mediante agitación mecánica (700 rpm.), utilizando sulfato de cobre pentahidratado $\mathrm{CuSO}_{4} \cdot 5 \mathrm{H}_{2} \mathrm{O}$ y sulfato de níquel hexahidratado $\mathrm{NiSO}_{4} \cdot 6 \mathrm{H}_{2} \mathrm{O}$ de $99 \%$ de pureza, ambos marca Fluka, en $200 \mathrm{~mL}$ de agua desmineralizada. A partir del efluente concentrado se obtuvieron soluciones de concentración inicial $5 \mathrm{mg} / \mathrm{L}$. Para la regulación del $\mathrm{pH}$ del efluente se prepararon soluciones $1 \mathrm{~N}$ de hidróxido de sodio $(\mathrm{NaOH})$ y ácido clorhídrico $(\mathrm{HCl})$, ambos de calidad analítica.

\subsection{Ensayos}

En los ensayos se usaron las variables que principalmente afectan la adsorción de metales por biosorbentes, tales como $\mathrm{pH}$, concentración del bisorbente y tiempo de contacto. Estos parámetros se usaron en el rango más comúnmente aplicados, basados en la precipitación de los iones cobre $(\mathrm{pH}$ superior a 6), tiempos para alcanzar el equilibrio de adsorción de iones, concentración más común del metal en efluentes y concentraciones de biosorbentes que permitiera compara con resultados de referencia bibliográfica.

\subsubsection{Pruebas en lote ("batch")}

Para determinar la capacidad de biosorción de las cáscaras de maní se hizo ensayos en sistemas en lote. Los ensayos se realizaron en vasos de precipitados de $600 \mathrm{~mL}$, mezclando el sorbente con el efluente mediante un agitador magnético marca Nova a una velocidad de $700 \mathrm{rpm}$. El pH fue ajustado con una solución de $\mathrm{NaOH}$ o $\mathrm{HCl}$, y las mediciones se realizaron en un pH-metro marca Hanna. Los ensayos se efectuaron a temperatura ambiente $\left(18-23^{\circ} \mathrm{C}\right)$. Una vez efectuada la remoción se realizó una etapa de separación sólido-líquido utilizando papel filtro Advantec №5 B, además se usó porta embudos y embudos de vidrio. Posteriormente, se midió el pH final de la solución filtrada y se tomó una muestra de aproximadamente $50 \mathrm{~mL}$ para análisis químico de cobre y níquel.

\subsubsection{Adsorción de iones cobre y níquel}

Mediante la combinación de variables y utilizando la solución preparada, según la metodología descrita, se realizaron pruebas en lote para la remoción de cobre y la remoción de níquel, considerando las siguientes variables y rangos: $\mathrm{pH}$ inicial del efluente (2 a 6), tiempo de contacto (5 a 60 minutos), concentración del biosorbente (10 a $60 \mathrm{~g} / \mathrm{L})$ y concentración inicial del metal en el efluente (cobre: 9,1 a 980,8 mg/L; níquel: 9,67 a 898,0 mg/L). Además, se realizó un ensayo para evaluar la remoción simultánea de iones cobre y níquel, bajo condiciones de referencia obtenidas en el sistema monocomponente y utilizando una solución de concentración inicial de $5 \mathrm{mg} / \mathrm{L}$ de cobre y de $5 \mathrm{mg} / \mathrm{L}$ de níquel. 


\section{RESULTADOS}

\subsection{Efecto del pH de la solución}

La Figura 1 muestra los resultados de la remoción de iones cobre y níquel por el biosorbente, en un intervalo de $\mathrm{pH}$ entre 2 y 6 . Se observa que a pH 2, la remoción de cobre fue $25 \%$, mientras que la remoción de níquel es nula, la remoción fue menor y estadísticamente diferente de los valores obtenidos a pH mayor. Este resultado se debe probablemente a que la elevada concentración de iones $\mathrm{H}^{+}$en este $\mathrm{pH}$ puede competir con los cationes por la adsorción en los sitios activos de la biomasa. La remoción de los iones cobre y níquel sobre $\mathrm{pH} 3$, fue estadísticamente similar, por lo que se continuó los experimentos de este estúdio a pH 5, en atención a que la mayoría de los autores que han trabajado con cascaras de maní han informado que en este $\mathrm{pH}$ se alcanza la mayor remoción de estos iones.

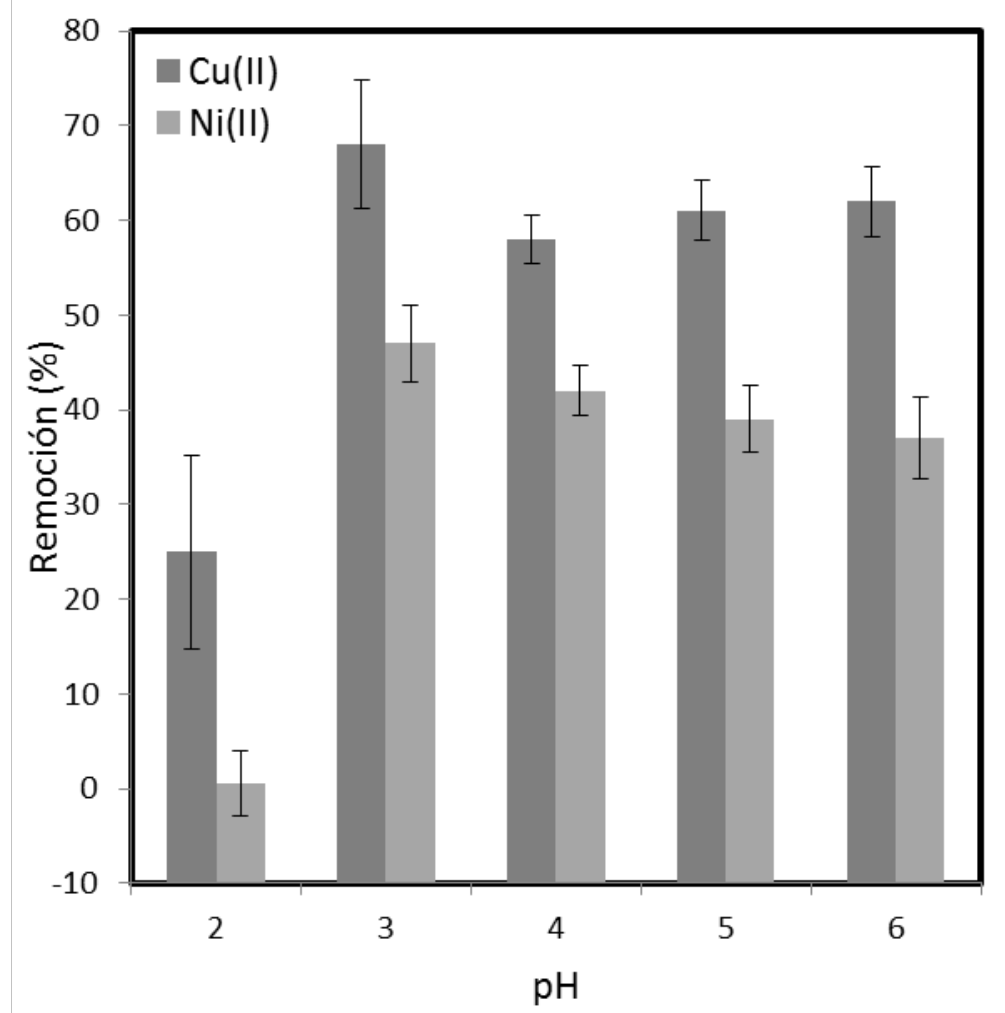

Figura 1: Efecto del pH sobre la remoción de iones cobre y níquel por las cáscaras de maní (tiempo de contacto de $30 \mathrm{~min}$; velocidad de agitación de 700 rpm; concentración del biosorbente 20g/L; concentración de metal en la solución $5 \mathrm{mg} / \mathrm{L}$ ). 


\subsection{Efecto del tiempo de contacto}

El efecto del tiempo de contacto sobre la remoción de iones cobre y níquel se muestra en la Figura 2. Las pruebas se realizaron variando el tiempo entre 5 y 60 minutos. De los resultados obtenidos se observa que la adsorción de iones níquel lograda a 15 minutos alcanza el 43\%, al incrementarse el tiempo de contacto se obtiene un $46 \%$ de remoción a los 60 minutos. La remoción de iones cobre presenta claramente el mejor rendimiento, apreciándose que 30 minutos es un tiempo suficiente para alcanzar una máxima remoción del orden de $65 \%$.

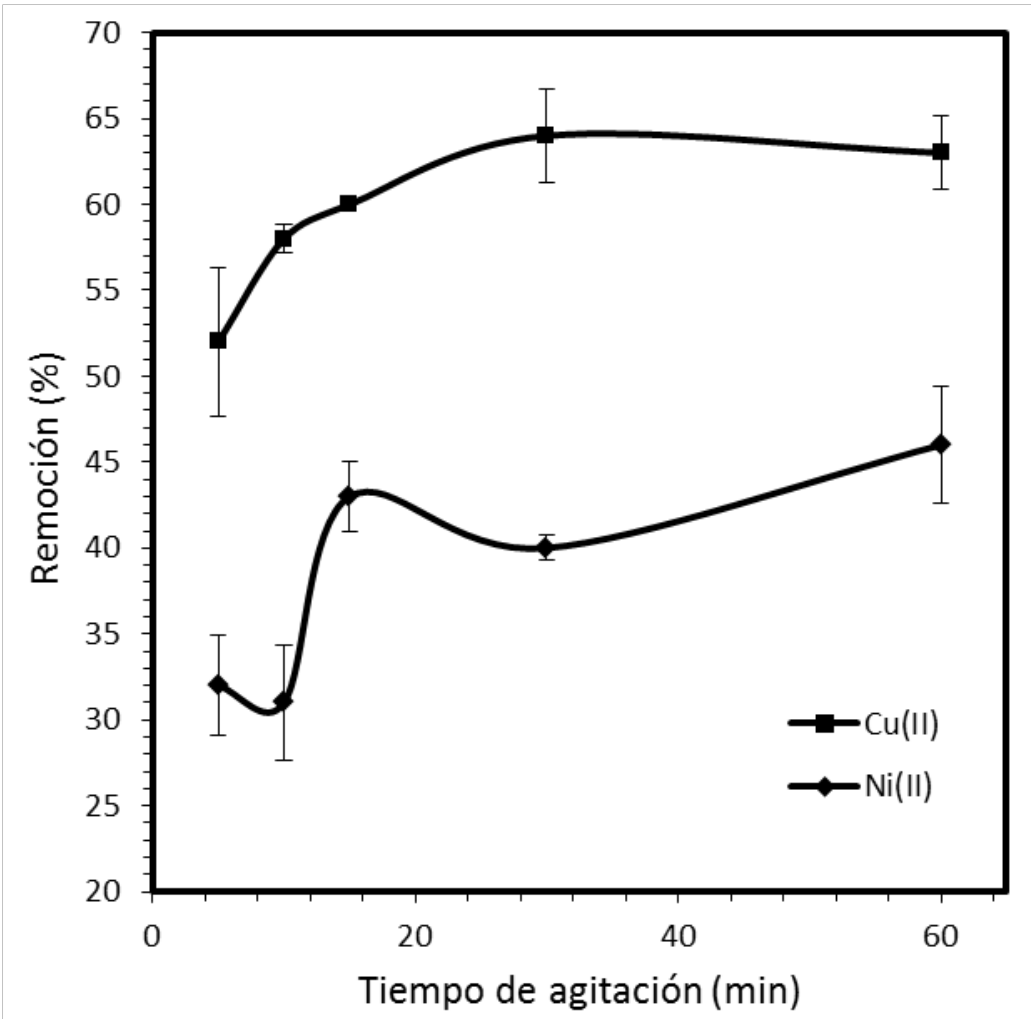

Figura 2: Efecto del tiempo de agitación sobre la remoción de iones cobre y níquel (velocidad de agitación 700 rpm; concentración del biosorbente $20 \mathrm{~g} / \mathrm{L}$; concentración inicial de metal en la solución 5 mg/L; pH 5).

\subsection{Efecto de la concentración del biosorbente}

El efecto de la concentración del biosorbente sobre la remoción de cobre y níquel se presenta en la Figura 3. Las pruebas se realizaron variando la concentración del biosorbente de 10 a $60 \mathrm{~g} / \mathrm{L}$. Se observó que con el aumento de la concentración del biosorbente, la eliminación de ambos iones no varió estadísticamente ( $p=0,5$; test $T$ de Student). La remoción de iones níquel fue menor que las de iones cobre en todo el intervalo estudiado. De acuerdo a lo anterior, se trabajó con una concentración de $20 \mathrm{~g} / \mathrm{L}$ de biosorbente para ambos casos. Altun y Pehlivan (2007) utilizando cáscara de nuez-almendra y aserrín, obtuvieron remoción de cobre similar a la determinada en este trabajo para igual concentración de biosorbente. 


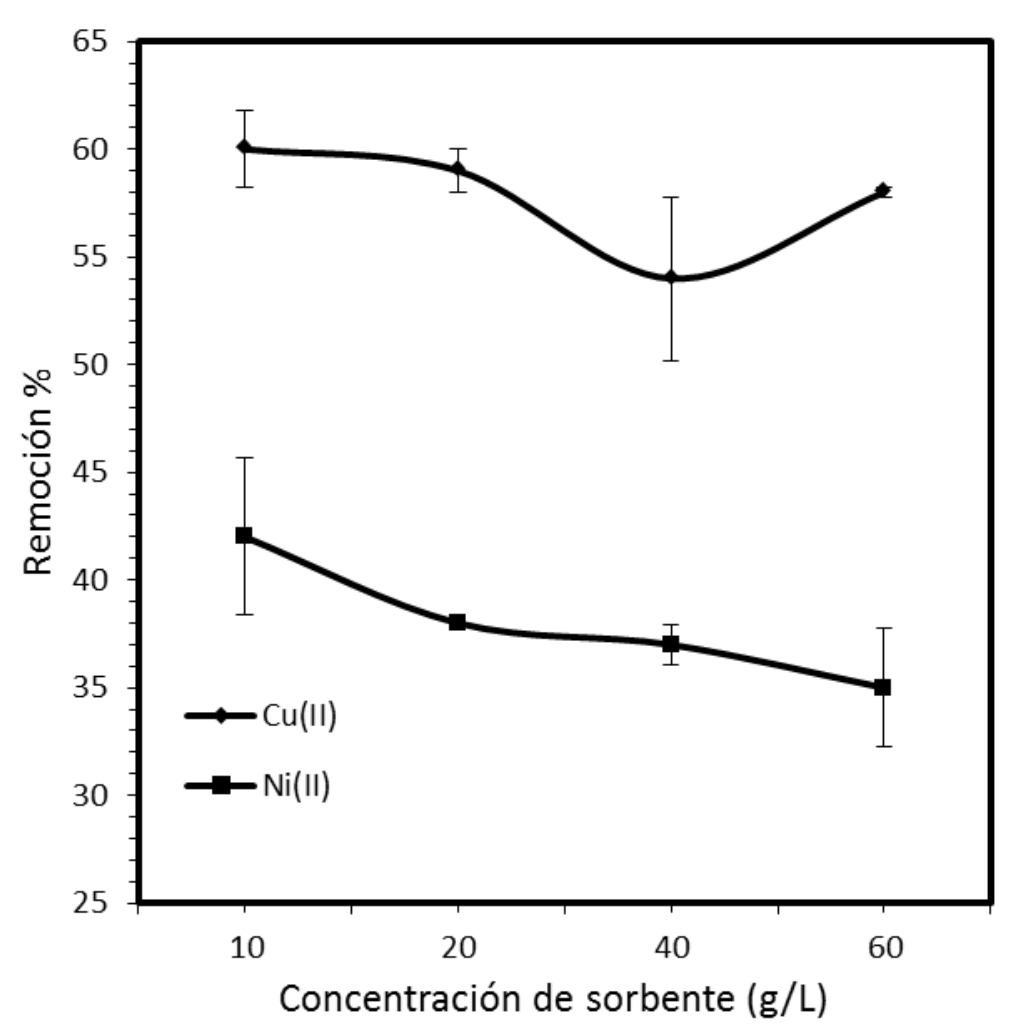

Figura 3: Efecto de la concentración del biosorbente sobre la remoción de iones cobre y iones níquel (velocidad de agitación de 700 rpm, tiempo de contacto de 15 minutos; concentración de metal en la solución 5 mg/L; pH 5).

\subsection{Efecto de la concentración inicial de cobre y níquel en la solución}

Para conocer la captación de iones cobre y níquel por el biosorbente, se realizaron experimentos variando la concentración inicial de cobre de $9,1 \mathrm{mg} / \mathrm{L}$ a $980,8 \mathrm{mg} / \mathrm{L}$ y la concentración inicial de níquel de $9,67 \mathrm{mg} / \mathrm{L}$ a $898,0 \mathrm{mg} / \mathrm{L}$. En la Figura 4 se presenta la captación de iones cobre y níquel por el biosorbente, en función de la concentración inicial de estos iones. Para ambos casos se observó un notorio aumento en la captación de iones hasta llegar a los $150 \mathrm{mg} / \mathrm{L}$, posteriormente disminuye la captación. Se aprecia para ambos iones que en el intervalo de concentración inicial de 2 a $150 \mathrm{mg} / \mathrm{L}$ existe una relación directa entre la concentración de iones y la captación de éstos, es decir al aumentar la concentración de iones también crece la adsorción, sin embargo a una concentración cercana a los $1000 \mathrm{mg} / \mathrm{L}$ de estos iones se observa una disminución en la captación de iones, principalmente de cobre. 


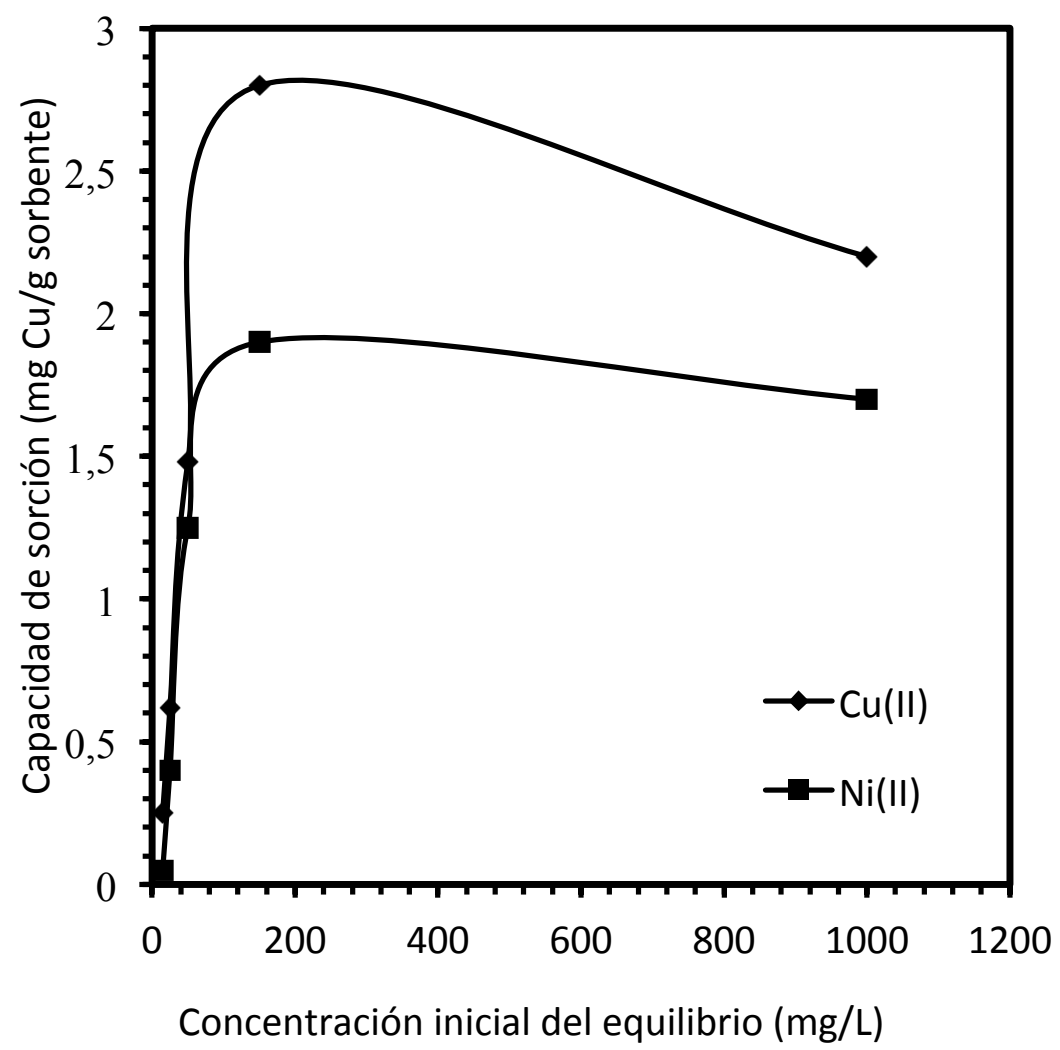

Figura 4: Efecto de la concentración inicial de iones cobre y níquel sobre la captación de iones por las cáscaras de maní (tiempo de contacto 30 minutos; concentración del biosorbente $20 \mathrm{~g} / \mathrm{L}$ ).

\subsection{Efecto de la remoción simultánea de iones cobre y níquel}

En la remoción simultánea de la mezcla de iones $\mathrm{Cu}$ (II) y Ni (II), la remoción de níquel obtenida, $45,70 \%$, fue inferior a la del cobre, 69,91 \%. Los resultados alcanzados previamente en forma individual marcaron la misma tendencia para la variable $\mathrm{pH}$, tiempo contacto y concentración de adsorbente, donde en promedio se obtuvieron remociones del orden de 40,3\% y 61,63\% para níquel y cobre respectivamente. Se apreció un leve aumento en la remoción de los iones cuando ambos estaban presentes en la solución, esto indicaría que al estar ambos iones en la solución existiría una interacción entre ellos lo que permitiría potenciar la adsorción en las cáscaras de maní.

\section{DISCUSIÓN}

El pH de la solución es uno de los parámetros más críticos en la remoción de iones metálicos desde soluciones acuosas; está relacionado directamente con la competencia entre iones del metal y los iones hidrógeno por los sitios activos del sorbente (Kumar et al. 2011). Varios investigadores han informado el efecto del $\mathrm{pH}$ sobre la remoción de iones cobre utilizando diferentes desechos agrícolas, tales como cáscara de avellana (Demirbas, Karadag, Alkan y Dogan, 2008), corteza de álamo (Dundar, Nuhoglu y Nuhoglu, 2008), cáscara de nuez (Kumar, Ramalingam, Sathyaselvabala, 
Kirupha y Sivanesan, 2011), cáscara de avellana y almendra (Altun y Pehlivan, 2007), residuo de manzana (Lee y Yang, 1997), cáscara de castaña (Yao, Qi y Wang, 2010), residuos de oliva (Pagnanelli, Mainelli, Veglio y Toro, 2003; Pagnanelli, Mainelli, De Angelis y Toro, 2005; Tapia, Santander, Pavez, Valderrama, Guzmán y Romero, 2011) y cáscara de maní (Zhu, Wang y Chen, 2009; Tapia, 2002; Oliveira, Paula, Freitas y Figueiredo, 2009), estos investigadores indican que el intervalo de $\mathrm{pH}$ donde ocurren las máximas remociones de cobre varía entre 4 a 5 . Por otro lado, respecto al níquel, la literatura informa que utilizando algas café (Basha, Murthy y Jha, 2009), Rhizopus arrhizus (Sag, Kaya y Kutsal, 2000) y tallos de desecho de uva (Villaescusa, Fiol, Martínez, Miralles, Poch y Serarols, 2004), la remoción máxima de níquel ocurre a un valor de pH 5. Por otro lado, Oliveira et al. (2010) informaron que el pH entre 5 y 6 era el más favorable para la remoción de iones cobre, níquel y zinc, no obstante, estos investigadores observaron que la capacidad de sorción aumentó fuertemente para $\mathrm{pH}$ en el intervalo 3-4, y que para $\mathrm{pH}$ superiores a 4 el cambio en los valores de capacidad no era significativo para cobre y níquel. Zhu et al. (2009) explicaron que lo señalado anteriormente ocurría porque el punto de carga cero para las cáscaras de maní se producía en $\mathrm{pH}$ aproximadamente 3,5. En el presente trabajo, el análisis estadístico realizado indicó que estadísticamente no hubo variación de la remoción de iones cobre y níquel con el aumento del pH, desde 3 a 6. En atención a lo anterior, se utilizó pH 5 en las diferentes pruebas, ya que permitiría comparar resultados con otros autores.

La adsorción de los iones cobre y níquel en las cáscaras de maní es dependiente del tiempo de contacto entre el adsorbente y los iones, es común que esta adsorción ocurra en los primeros minutos de contacto; llegándose a un tiempo de equilibrio, a partir del cual la remoción de los iones se torna lenta. Diferentes tiempos de equilibrio en el proceso de biosorción de cobre y níquel se ha informado en la literatura, no obstante varios investigadores han informado valores concordantes con el tiempo obtenido en el presente trabajo. Oliveira et al. (2009) indicaron que la mayor parte de la remoción de cobre y plomo en la biosorción con cáscaras de maní, ocurría en los primeros 20 minutos de contacto, mostrando en ese tiempo una buena tasa de captación de iones. Por otra parte, los experimentos cinéticos realizados por Olivera et al. (2010), mostraron que la mayor parte de la adsorción de iones cobre, níquel y zinc en cáscara de maní se producía en los primeros 30 minutos. Kumar et al. (2011) usando cáscaras de nuez obtuvieron una remoción de cobre del orden del $70 \%$ en un tiempo de equilibrio de biosorción también en 30 minutos, por otra parte, Basha et al. (2009) utilizando como sorbente algas marinas en la remoción de níquel lograron la mayor remoción de estos iones igualmente a los 30 minutos. Hawari y Mulligan (2006) utilizando gránulos anaeróbicos como biosorbente para la eliminación de plomo, cobre, cadmio y níquel desde soluciones acuosas, informaron que en la captación de estos iones metálicos el equilibrio de adsorción se alcanzaba en un tiempo del orden de 30 minutos. Estos antecedentes pueden indicar que 30 minutos sería el tiempo adecuado para alcanzar una remoción satisfactoria, lo que coincide con el resultado obtenido en el presente trabajo.

En relación al trabajo realizado en sistemas de más de un componente (cobre y níquel), resultados concordantes con el presente estudio fueron informados por Olivera et al. (2010), quienes indicaron que las cáscaras de maní tenían mayor afinidad por cobre que por níquel y zinc cuando ambos están presentes (sistema tricomponente). Por otra parte, Villaescusa et al. (2004) utilizando tallos de uva como biosorbentes obtuvieron remociones simultáneas de iones cobre y 
níquel del orden del $70 \%$, a partir de soluciones con una concentración inicial a $10 \mathrm{mg} / \mathrm{L}$ y tiempo de contacto 30 minutos.

\section{CONCLUSIONES}

Los resultados experimentales obtenidos en el presente trabajo permiten deducir las siguientes conclusiones:

En los ensayos realizados en el sistema en lote se alcanzaron remociones entre 3,6 \% y 47,6\% para los iones níquel y 4,3 \% y 68,3 \% para iones cobre. La máxima remoción de los iones se alcanzó en $\mathrm{pH} 3$.

La cinética de biosorción se produce en forma rápida durante los primeros 15 minutos, logrando eliminaciones cercanas a $60,0 \%$ para el cobre y $43,0 \%$ para el níquel. A tiempos mayores en ambos casos no se logra incrementar significativamente la remoción de cobre, los valores máximos se alcanzan en un tiempo de 30 minutos (cobre) y 60 minutos (níquel).

A medida que aumenta la concentración del biosorbente la eliminación de iones cobre y níquel sufre una leve disminución. La remoción de iones níquel es menor a la eliminación de los iones cobre, en todo el rango estudiado. De acuerdo a los resultados obtenidos, se opta por una concentración de $20 \mathrm{~g} / \mathrm{L}$ de biosorbente como la más adecuada para ambos casos.

En relación a la captación de iones cobre y níquel por el biosorbente en función de la concentración inicial de estos iones, se observa un aumento notorio en la captación de iones hasta llegar a una concentración de $150 \mathrm{mg} / \mathrm{L}$. Para ambos iones en el intervalo de concentración inicial de 2 a $150 \mathrm{mg} / \mathrm{L}$ existe una relación directa entre la concentración de iones y la captación de éstos, es decir al aumentar la concentración de iones también crece la adsorción. Sin embargo, a mayor concentración inicial de cobre y níquel disminuye la captación de iones, principalmente de cobre.

En las pruebas de remoción simultánea de la mezcla de iones $\mathrm{Cu}(\mathrm{II})$ y $\mathrm{Ni}(\mathrm{II})$, se observa que la remoción de níquel $(45,7 \%)$ es inferior a la del cobre (69,91 \%). Estos resultados marcaron la misma tendencia observada en las pruebas de remoción individual realizadas con soluciones de iones cobre y níquel, para las variables $\mathrm{pH}$, tiempo de contacto y concentración del adsorbente.

\section{REFERENCIAS}

Acheampong M.A., Pereira J.P.C., Meulepas R.J.W., y Lens P.N.L. (2012). Kinetics modeling of Cu(II) biosorption on to coconut shell and Moringa oleifera seeds from tropical regions. Environmental Technology, 33(4), 409-417.

Altun, T., y Pehlivan E. (2007). Removal of copper(II) ions from aqueous solutions by walnut, hazelnut and almond shells. CLEAN - Soil, Air, Water, 35(6), 601-606. 
Areco M.M., Saleh-Medina L., Trinelli M.A., Marco-Brown J.L., y Dos Santos, A.M. (2013). Adsorption of $\mathrm{Cu}(\mathrm{II}), \mathrm{Zn}(\mathrm{II}), \mathrm{Cd}(\mathrm{II})$ and $\mathrm{Pb}$ (II) by dead Avena fatua biomass and the effect of these metals on their growth. Colloids and Surfaces B Biointerfaces, 110, 305-312.

Basha S., Murthy Z.V.P., y Jha B. (2009). Removal of $\mathrm{Cu}(\mathrm{II})$ and $\mathrm{Ni}(\mathrm{II})$ from industrial effluents by brown seaweed Cystoseira indica. Industrial \& Engineering Chemistry Research, 48(2), 961 975.

Candan M., Tay F., Avan I., y Tay T. (2017). Removal of copper(II) and nickel(II) ions from aqueous solution using non-living lichen Ramalina fraxinea biomass: investigation of kinetics and sorption isotherms. Desalination and Water Treatment, 75, 148-157.

Chen Z., Ma W., y Han M. (2008). Biosorption of nickel and copper onto treated alga (Undaria pinnatifida): Application of isotherm and kinetic models. Journal of Hazardous Materials, 155(1-2), 327-333.

Chigondo F., Nyamunda B.C., Sithole S.C., y Gwatidzo L. (2013). Removal of lead (II) and copper (II) ions from aqueous solution by baobab (Adononsia digitata) fruit shells biomass. Journal of Applied Chemistry, 5(1), 43-50.

Demirbas O., Karadag A., Alkan M., y Dogan M. (2008). Removal of copper ions from aqueous solution by hazelnut shell. Journal of Hazardous Materials, 153(1-2), 677-684.

Dundar M., Nuhoglu C., y Nuhoglu Y. (2008). Biosorption of $\mathrm{Cu}(\mathrm{II})$ ions onto the litter of natural trembling poplar forest. Journal of Hazardous Materials, 151(1), 86-95.

Hawari A.A., y Mulligan C.N. (2006). Biosorption of lead(II), cadmium(II), Copper(II) and nickel(II) by anaerobic granular biomass. Bioresource Technology, 97(4), 692-700.

Khan A.M., Ahmad C. S., Farooq U., Mahmood K., Sarfraze M., Balkhair K.S., y Ashraf M.A. (2015). Removal of metallic elements from industrial waste water through biomass and clay. Frontiers in Life Science, 8(3), 223-230.

Kondo, K., Sumi, H., y Matsumoto, M. (1996). Adsorption characteristics of metal ions on chitosan chemically modified by D-galactose. Separation Science and Technology, 31(12), 1771-1775.

Kumar P.S., Ramalingam S., Abhinaya R.V., Kirupha S.D., Murugesan A., y Sivanesan S. (2012). Adsorption of metal ions the chemically modified agricultural waste. CLEAN - Soil, Air, Water, 40(2), 188-197.

Kumar P.S., Ramalingam S., Sathyaselvabala V., Kirupha S.D., y Sivanesan S. (2011). Removal of copper(II) ions from aqueous solution by adsorption using cashew nut shell. Desalination, 266, 63-71.

Lee S., y Yang J. (1997). Removal of copper in aqueous solution by apple wastes. Separation Science and Technology, 32(8), 1371-1387.

Oliveira F.D., Soares A.C., Freitas O.M., y Figueiredo S.A. (2010). Copper, nickel and zinc removal by peanut hulls: Batch and column studies in mono, tri-component systems and with real effluent. Global NEST Journal, 12(2), 206-214.

Oliveira F.D., Paula J.H., Freitas O.M., y Figueiredo S.A. (2009). Copper and lead removal by peanut hulls: Equilibrium and kinetic studies. Desalination, 248 (1-3), 931-940.

Pagnanelli F., Mainelli S., De Angelis S., y Toro L. (2005). Biosorption of protons and heavy metal sonto olive pomace: Modelling of competition effects. Water Research, 39 (8), 1639-1651.

Pagnanelli F., Mainelli S., Veglio F., y Toro L. (2003). Heavy metal removal by olive pomace: biosorbent characterisation and equilibrium modelling. Chemical Engineering Science, 58(20), 4709-4717. 
Sag Y., Kaya A., y Kutsal T. (2000). Biosorption of lead(II), nickel(II) and copper(II) on Rhizopus arrhizus from binary and ternary metal mixtures. Separation Science and Technology, 35(16), 2601-2617.

Singh J., Ali A., y Kumar R. (2013). Removal of $\mathrm{Ni}^{2+}, \mathrm{Cu}^{2+}$ and $\mathrm{Zn}^{2+}$ using different agricultural residues: Kinetics, isotherm modeling and mechanism via chemical blocking. Asian Journal of Chemistry, 25(11), 6377-6386.

Tapia P., Santander M., Pavez O., Valderrama L., Guzmán D., y Romero L. (2011). Biosorción de iones cobre con biomasa de algas y orujos deshidratados. Revista de Metalurgia, 47(1), 15-28.

Tapia P. (2002). Estudio de materiales orgánicos e inorgánicos en la remoción de iones metálicos. Tesis de Magíster en Metalurgia, Universidad de Atacama, Copiapó, Chile, 135 pp.

Villaescusa I., Fiol N., Martínez M., Miralles N., Poch J., y Serarols J. (2004). Removal of copper and nickel ions from aqueous solution by grape stalks wastes. Water Research, 38(4), 992-1002.

Yao Z.Y., Qi J.H., y Wang L.H. (2010). Equilibrium, kinetic and thermodynamic studies on the biosorption of $\mathrm{Cu}(\mathrm{II})$ onto chestnut shell. Journal of Hazardous Materials, 174(1-3), 137-143.

Zhu C.H., Wang L.P., y Chen W. (2009). Removal of $\mathrm{Cu}(\mathrm{II})$ from aqueous solution by agricultural byproduct: Peanut hull. Journal of Hazardous Materials, 168(2-3), 739-746. 